\title{
Demonstration by Light and Electron Microscopy of Capsules on Gonococci Recently Grown in vivo
}

\author{
By RAQUEL DEMARCO DE HORMAECHE AND \\ MARGARET J. THORNLEY \\ Immunology Division, Department of Pathology, University of Cambridge, \\ Cambridge CB2 $2 Q Q$ \\ AND AUDREY M. GLAUERT \\ Strangeways Research Laboratory, Cambridge
}

(Received 26 August 1977; revised 15 December 1977)

\begin{abstract}
A study by light microscopy, using Leishman's stain alone or Leishman's stain followed by nigrosin, showed the presence of capsules on gonococci of two strains subcultured from subcutaneous chambers in guinea pigs. With the Alcian blue method of preparation for electron microscopy, gonococci of both strains recently grown in vivo showed densely stained capsules on some cells, while others in the same preparation showed only irregular masses of dense material on their surfaces with strands connecting adjacent bacteria. Treatment with antiserum, complement and conglutinin revealed irregular masses and strands of extracellular material with fixatives that did not contain Alcian blue.
\end{abstract}

\section{INTRODUCTION}

Although gonococci are classically described as non-capsulated Gram-negative diplococci, the presence or absence of capsules in these micro-organisms has been a matter of discussion for many years. Recently Richardson \& Sadoff (1977) have described a 'capsulelike effect' in recently isolated gonococci studied by the India ink method. James \& Swanson (1977) have convincingly demonstrated the presence of capsules on gonococci by light microscopy using a variety of staining methods, and Hendley et al. (1977) have shown capsules by the electron microscopy of antiserum-treated gonococci.

In this study we have examined strains recently grown in vivo, in guinea-pig chambers, by light microscopy to investigate whether capsules are detectable. For electron microscopy, we have also used fixation methods which have previously revealed capsules and extracellular material on lactobacilli growing in vivo (Brooker \& Fuller, 1975).

\section{METHODS}

Strains of gonococci. These were isolated from samples kindly provided by the Genito-Medical Clinic, Addenbrooke's Hospital, and the Public Health Laboratory, Cambridge. Two strains of gonococci were selected for this study, both from acute cases of gonorrhoea: Gc12 was isolated from a vaginal sample and Gc40 from urine of a male patient. Both were confirmed as Neisseria gonorrhoeae by Gram staining, oxidase reaction and oxidation of sugars. The cultures were suspended in Greaves medium (Greaves, 1960) and frozen in liquid nitrogen (Ward \& Watt, 1971) for storage.

In vitro subcultures were made on Difco Gc agar base enriched with $1 \%(\mathrm{v} / \mathrm{v}) \mathrm{BBL}$ IsoVitaleX (referred to as $\mathrm{GcIv}$ ), and were incubated at $37^{\circ} \mathrm{C}$ in candle jars.

In vivo growth of gonococci was obtained using subcutaneous plastic chambers (Veale et al., 1975). Polyallomer $4 \mathrm{ml}$ centrifuge tubes perforated in several places with a hot cork-borer and closed at the end 
by pressure under heat were used as chambers. The chambers were sterilized in an autoclave. Two chambers were implanted under the dorsal skin of each guinea pig ( 400 to $500 \mathrm{~g}$ ). Three months were allowed for

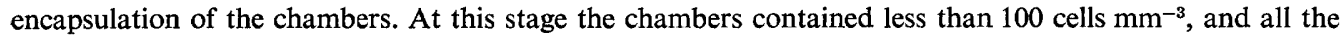
cells were macrophages.

Gonococci for inoculation were not selected on the basis of colony type. Cultures were grown on GcIv agar for 22 to $24 \mathrm{~h}$ and then suspended in saline to give approximately $10^{8}$ gonococci in $0.1 \mathrm{ml}$. This volume was inoculated into each guinea-pig chamber, and dilutions were plated to check the inoculum size. Fluid was withdrawn from the chambers weekly and subcultured directly on to GcIv agar, this being regarded as the first subculture.

Colonial morphology. This was observed on the first subculture from the guinea-pig chamber, using a Zeiss Stereo-microscope II with both transmitted light and incident light at an angle of approximately $45^{\circ}$.

Light microscopy. For Gram staining, specimens were treated with $2 \%(\mathrm{w} / \mathrm{v})$ crystal violet followed by Lugol's iodine, each for $30 \mathrm{~s}$, decolorized with either acetone or acetone/ethanol $(1: 5, \mathrm{v} / \mathrm{v})$ and counterstained with $0.5 \%(\mathrm{w} / \mathrm{v})$ safranine for $2 \mathrm{~min}$.

For capsule staining, gonococci of the first subculture were suspended on the slide in $0.9 \%(w / v)$ sodium chloride containing $100 \mu \mathrm{gDNAase} \mathrm{ml}^{-1}$ and smeared gently and thinly for rapid drying. Dry, unfixed smears were stained with Leishman's stain for $2 \mathrm{~min}$, followed by Leishman stain diluted 1:3 with distilled water for $10 \mathrm{~min}$, and then washed with $0.2 \mathrm{M}$-Tris $/ \mathrm{HCl}$ buffer, $\mathrm{pH} \mathrm{6.8.} \mathrm{To} \mathrm{enhance} \mathrm{the} \mathrm{observation} \mathrm{of} \mathrm{capsules} \mathrm{a}$ small drop of $10 \%(\mathrm{w} / \mathrm{v})$ nigrosin was spread on the smears already stained with Leishman's stain.

Electron microscopy. (i) Normal fixation method (Thornley, Glauert \& Sleytr, 1973). Colonies were fixed in $2.5 \%(\mathrm{w} / \mathrm{v})$ glutaraldehyde in $0.09 \mathrm{M}$-cacodylate buffer, $\mathrm{pH} 7 \cdot 2$, containing $3 \mathrm{~mm}-\mathrm{CaCl}_{2}$, by adding a few drops of fixative to the agar surface bearing the colonies. After $5 \mathrm{~min}$ the colonies were removed and fixation was continued for $1 \mathrm{~h}$ at room temperature. After centrifugation, the pellets were washed in the cacodylate buffer overnight at $4{ }^{\circ} \mathrm{C}$, post-fixed with $1 \%(\mathrm{w} / \mathrm{v})$ osmium tetroxide in veronal acetate buffer for $1 \mathrm{~h}$ at $22{ }^{\circ} \mathrm{C}$, stained with $0.5 \%(\mathrm{w} / \mathrm{v})$ aqueous uranyl acetate for $1 \mathrm{~h}$ at $22{ }^{\circ} \mathrm{C}$, dehydrated in ethanol and embedded in Araldite.

(ii) Alcian blue method (Shea, 1971). Small portions of agar carrying colonies of gonococci grown for $18 \mathrm{~h}$ were placed in fixative consisting of $0.1 \mathrm{M}$-cacodylate buffer, $\mathrm{pH} 7 \cdot 2$, containing $3 \%(\mathrm{w} / \mathrm{v})$ glutaraldehyde and $0.5 \%(\mathrm{w} / \mathrm{v})$ Alcian blue. After $2 \mathrm{~h}$ at room temperature, the specimens were washed once in the cacodylate buffer, then treated for $2 \mathrm{~h}$ at room temperature in $0 \cdot 1 \mathrm{M}-s$-collidine buffer, $\mathrm{pH} 8 \cdot 0$, containing $1 \%(\mathrm{w} / \mathrm{v})$ osmium tetroxide and $1 \%(\mathrm{w} / \mathrm{v})$ lanthanum nitrate. This was followed by dehydration in ethanol and embedding in Araldite.

(iii) Pre-treatment of bacteria with anti-gonococcal or normal rabbit serum, complement and conglutinin. Anti-gonococcal serum was prepared in rabbits inoculated sequentially with a total of 14 strains of recently isolated gonococci, including Gc12, using a schedule based on that of Apicella \& Allen (1973). The antiserum was inactivated by heating at $56^{\circ} \mathrm{C}$ for $30 \mathrm{~min}$. For control preparations, inactivated normal rabbit serum was used in place of the antiserum. Serum from $\mathrm{a} \mathrm{C}_{6}$-deficient rabbit was used as a source of complement to avoid lysis of the gonococci. Inactivated bovine serum was used as a source of conglutinin.

Gonococci grown for $20 \mathrm{~h}$ on GcIv agar were suspended in complement fixation test diluent (CFT; Oxoid) containing $100 \mu \mathrm{g}$ DNAase $\mathrm{ml}^{-1}$ to a standard absorbance of 0.3 at $600 \mathrm{~nm}$. To $1 \mathrm{ml}$ of this suspension was added $1 \mathrm{ml}$ of either anti-gonococcal serum, diluted 1:10 in CFT, or an equal amount of normal rabbit serum, followed by $1 \mathrm{ml}$ of a 1:40 dilution in CFT of the $C_{6}$-deficient rabbit serum and $1 \mathrm{ml}$ of a 1:20 dilution of inactivated bovine serum. The preparation was mixed gently, and after $30 \mathrm{~min}$ at room temperature sufficient glutaraldehyde was added to give a final concentration of $2.5 \%(\mathrm{w} / \mathrm{v})$. Fixation for $30 \mathrm{~min}$ at room temperature was followed by centrifugation. The pellet was washed in cacodylate buffer overnight at $4{ }^{\circ} \mathrm{C}$, then treated as in the normal fixation method (above).

Comparisons were made between treatment with antiserum, complement and conglutinin, and treatment with antiserum only.

\section{RESULTS}

Many of the strains of $N$. gonorrhoeae isolated in our laboratory presented a distinctive appearance when stained by Gram's method. Some of the isolates had a pink background which left an unstained halo around the diplococci. This appearance, indicating the possible presence of capsules, was always seen in a few strains, such as Gc12, but only observed occasionally in others, such as Gc40. These two strains were therefore chosen for comparison in this study as representives of the two different staining patterns. 


\section{Colonial morphology}

No attempt was made on subculture to select any particular type of colony. After more than 30 subcultures in vitro, both strains gave rise to a mixture of colony types in which large fiat colonies, similar to types 3 and 4 of Kellogg et al. (1963), were clearly predominant. When bacteria grown for up to $80 \mathrm{~d}$ in a chamber in a guinea pig were subcultured, the predominant colony types still resembled types 3 and 4; some small convex colonies were also present but these represented only a very small proportion of the total (Figs 1 and 2).

\section{Light microscopy}

Leishman staining, with or without the addition of nigrosin, of Gc40 cells grown in vitro gave variable results in which some preparations showed capsules. The same strain subcultured once on GcIv from an infected guinea-pig chamber always showed clear haloes around the diplococci with either of the staining methods (Fig. 3). Strain Gc12 grown in vitro showed haloes when stained by the Leishman method, and this effect was enhanced by the addition of nigrosin (Fig. 4). After one subculture from the guinea-pig chamber the capsules were more evident in Leishman stain and were particularly clear after Leishman staining followed by addition of nigrosin; the cocci were often associated together in large clumps (Figs 5, 6 and 7).

With both strains, the first and third subcultures from the guinea-pig chamber gave indistinguishable results by light microscopy.

\section{Electron microscopy}

Gonococci at the third subculture after growth in vivo, which appeared clearly capsulated by light microscopy, were studied by electron microscopy. Different methods of preparation were compared to find the most effective treatment for revealing capsules.

Normal fixation. After normal fixation of colonies in situ, both strains showed well preserved cocci with many bacteria in the process of division (Fig. 8). At higher magnification (Fig. 9), the envelope of Gc40 had the appearance typical of Gram-negative bacteria (Glauert \& Thornley, 1969), with the plasma membrane (pm), dense layer (d), and outer membrane (om), which had a wavy outline. No traces of filaments or other extracellular material were seen in this preparation.

Alcian blue method. With the Alcian blue method, the outer membrane remained clearly visible (Figs 11 to 15 , om) although it was much smoother in outline than in cells fixed in the normal way (cf. Figs 9 and 12). Electron-dense material penetrated the outer membrane and formed a fairly even layer within the cell envelope between the outer membrane and the plasma membrane; the latter was difficult to distinguish. Mesosomes (Fig. 13, m) were heavily stained and, since they are believed to remain in connection with the plasma membrane, this observation is consistent with the idea that the plasma membrane is the barrier which prevents penetration of the electron-dense stain into the cytoplasm.

Some gonococci of both strains had layers of electron-dense material up to $50 \mathrm{~nm}$ thick outside the outer membrane (Figs 10, 11 and 15). Figure 10 shows a tetrad of Gc40, of which only three cells were within the plane of section. The dense layer appeared fairly uniform around one cell in this group (seen at higher magnification in Fig. 11) and was particularly thick near the region of division between cells (Fig. 11, arrow), while the layer was more patchy on an adjoining cell (Fig. 10, arrow). Many other gonococci in the preparation of $\mathrm{Gc} 40$ had little or no dense material adhering to the outer membrane except in isolated masses (Fig. 12, arrows), which were sometimes connected to strands (Fig. 12, s) of stained material stretching between cells. Similar strands were also seen in the spaces between cells (Figs 12 and 13). Figure 13 illustrates the contrast between adjoining cells with and without layers of extracellular dense material, and also the accumulation of dense material between cells. 

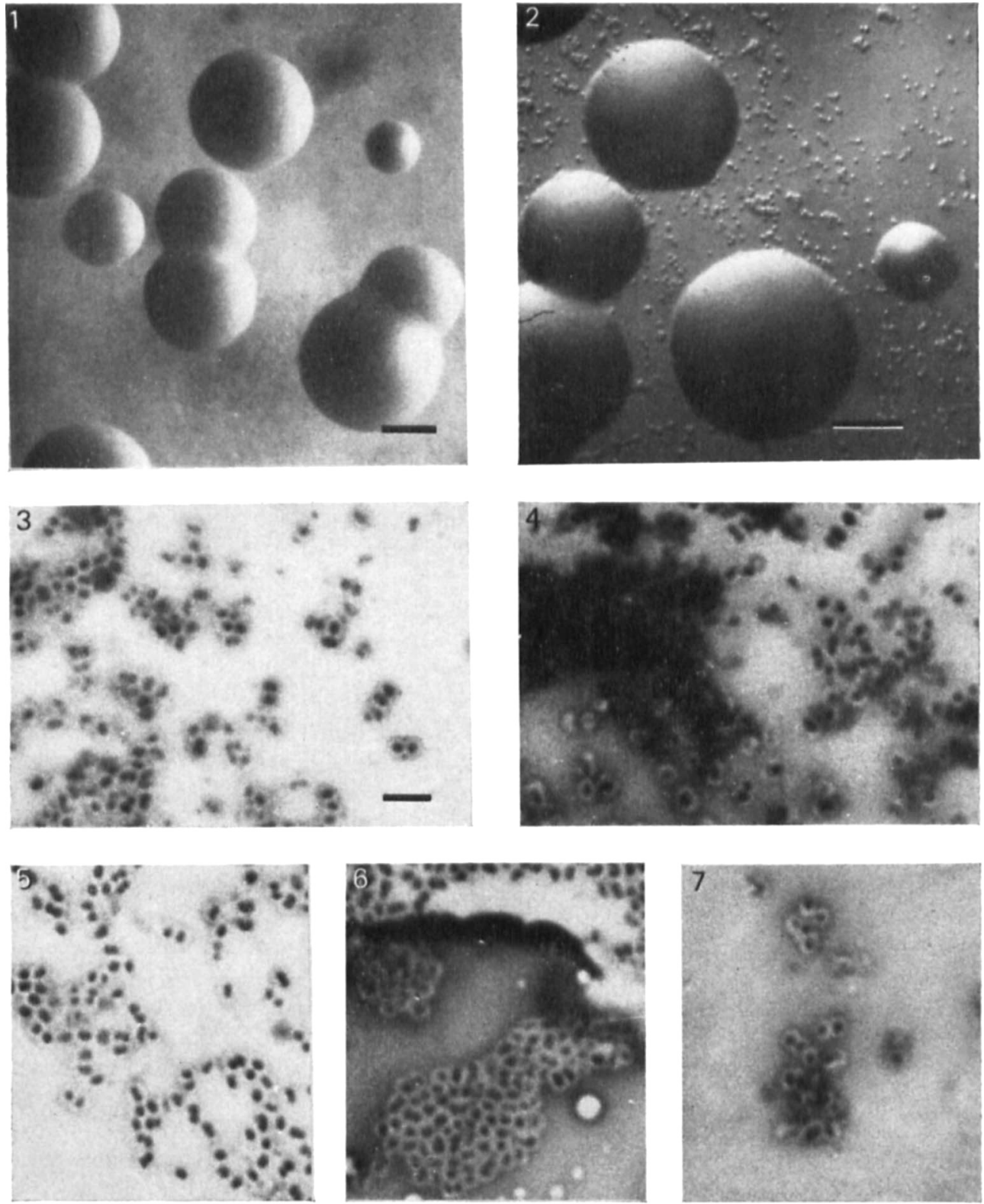

Figs 1, 2. Colonies of gonococci subcultured on GcIv from guinea-pig chambers infected for $80 \mathrm{~d}$. Bar markers represent $0.5 \mathrm{~mm}$.

Fig. 1. Gc40 shows predominantly large mucoid colonies. This field was chosen to include some small convex colonies, but these formed only a very small proportion of the whole.

Fig. 2. Gc12 also shows predominantly large flat colonies, with a few small conical colonies. Pus cells from the chamber fluid are seen on the surface of the agar and appear to cause small irregularities in the outlines of the colonies.

Figs 3 to 7. Light micrographs of gonococci, all at the same magnification; the bar marker on Fig. 3 represents $3 \mu \mathrm{m}$.

Fig. 3. Gc40 subcultured once from the guinea-pig chamber; all cells show distinct capsules. Leishman stain plus nigrosin.

Fig. 4. Gc12 subcultured many times in vitro; most cells have capsules. Leishman stain plus nigrosin.

Fig. 5. Gc12 subcultured once from the guinea-pig chamber; all cells are capsulated. Leishman stain.

Fig. 6. Gc12 subcultured once from the guinea-pig chamber; capsulated cells are in large clumps. Leishman stain plus nigrosin. The boundary of the nigrosin is included to show the enhanced contrast obtained.

Fig. 7. Gc12, prepared as in Fig. 6; capsules are clearly seen. 

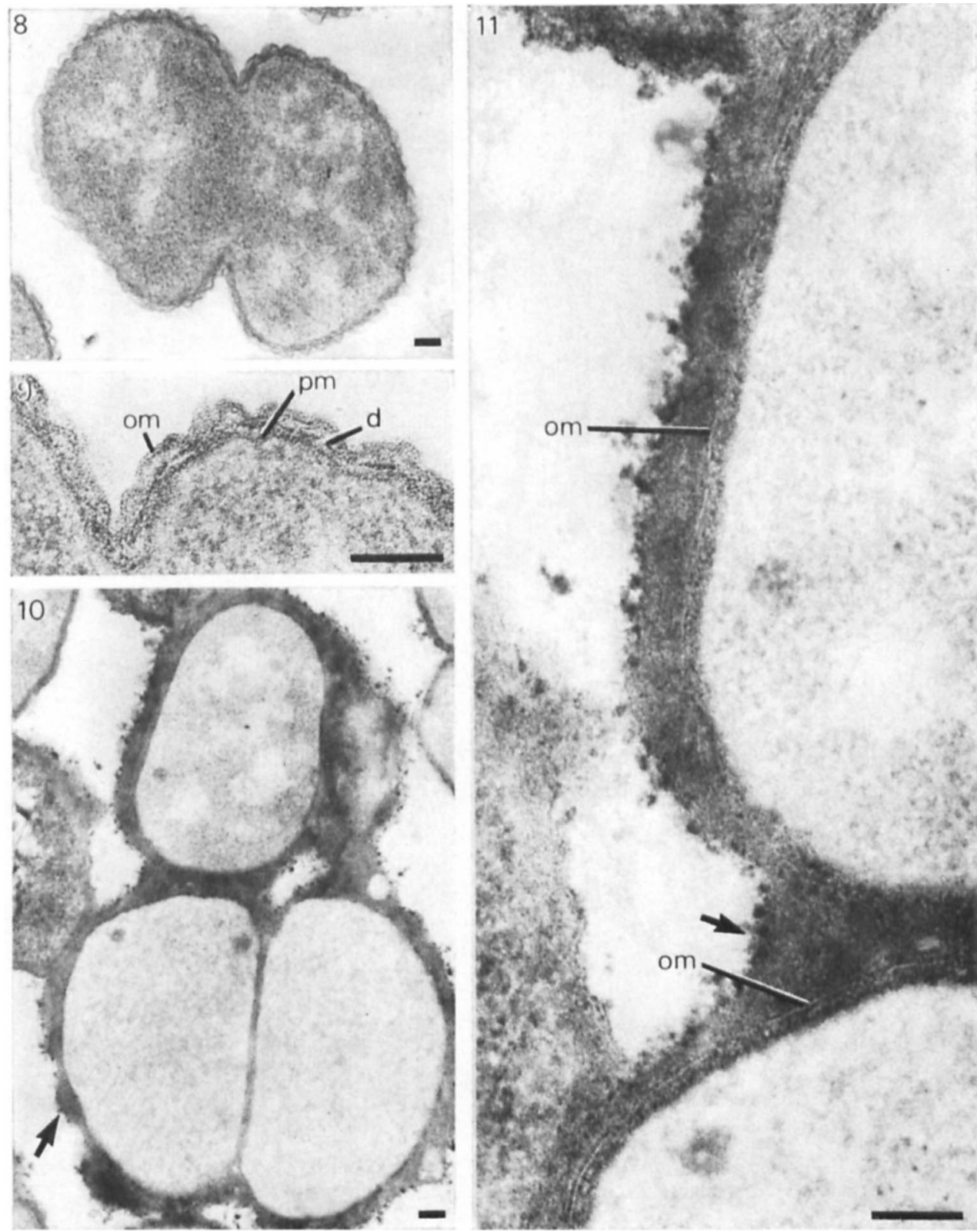

Figs 8 to 11. Electron micrographs of thin sections of gonococci (strain Gc40) at the third subculture after growth in vivo. Bar markers represent $0 \cdot 1 \mu \mathrm{m}$.

Fig. 8. After normal fixation; a well-preserved coccus in the process of division.

Fig. 9. Part of Fig. 8 at higher magnification; the bacterial envelope is seen to consist of the plasma membrane (pm), the dense layer (d) and the outer membrane (om), with no material visible outside the outer membrane.

Fig. 10. After Alcian blue treatment; a group of cells with a densely stained external layer that is continuous around one cell and irregular on another (arrow).

Fig. 11. Part of Fig. 10 at higher magnification; densely stained material forms a thick layer outside the outer membrane (om) and accumulates at the junction between the cells (arrow). 



Figs 12, 13. Electron micrographs of thin sections of gonococci (strain Gc40) at the third subculture after growth in vivo. Bar markers represent $0 \cdot 1 \mu \mathrm{m}$. Alcian blue preparations.

Fig. 12. Many cells have no dense material outside the outer membrane (om) except for irregular masses (arrows), sometimes connected to strands (s) stretching between cells.

Fig. 13. Adjoining cells vary greatly in the amount of dense material outside the outer membrane (om); s, strands; m, mesosome.

The Alcian blue preparation of Gc12 contained the same features as those seen in Gc40 but in different proportions. Thick continuous layers of extracellular material were found only rarely (Fig. 15), while the patchy distribution of dense material seen in Fig. 14 (arrows) was common. Strands (s) of dense material appeared to be continuous with either patches (Fig. 14) or layers (Fig. 15) of dense material attached to the outer membrane.

Zones of adhesion between adjoining cells were observed in this preparation (Fig. 14, z), and appeared similar to those described by Swanson, Kraus \& Gotschlich (1971) after treatment of gonococci with lanthanum alone with no Alcian blue. 

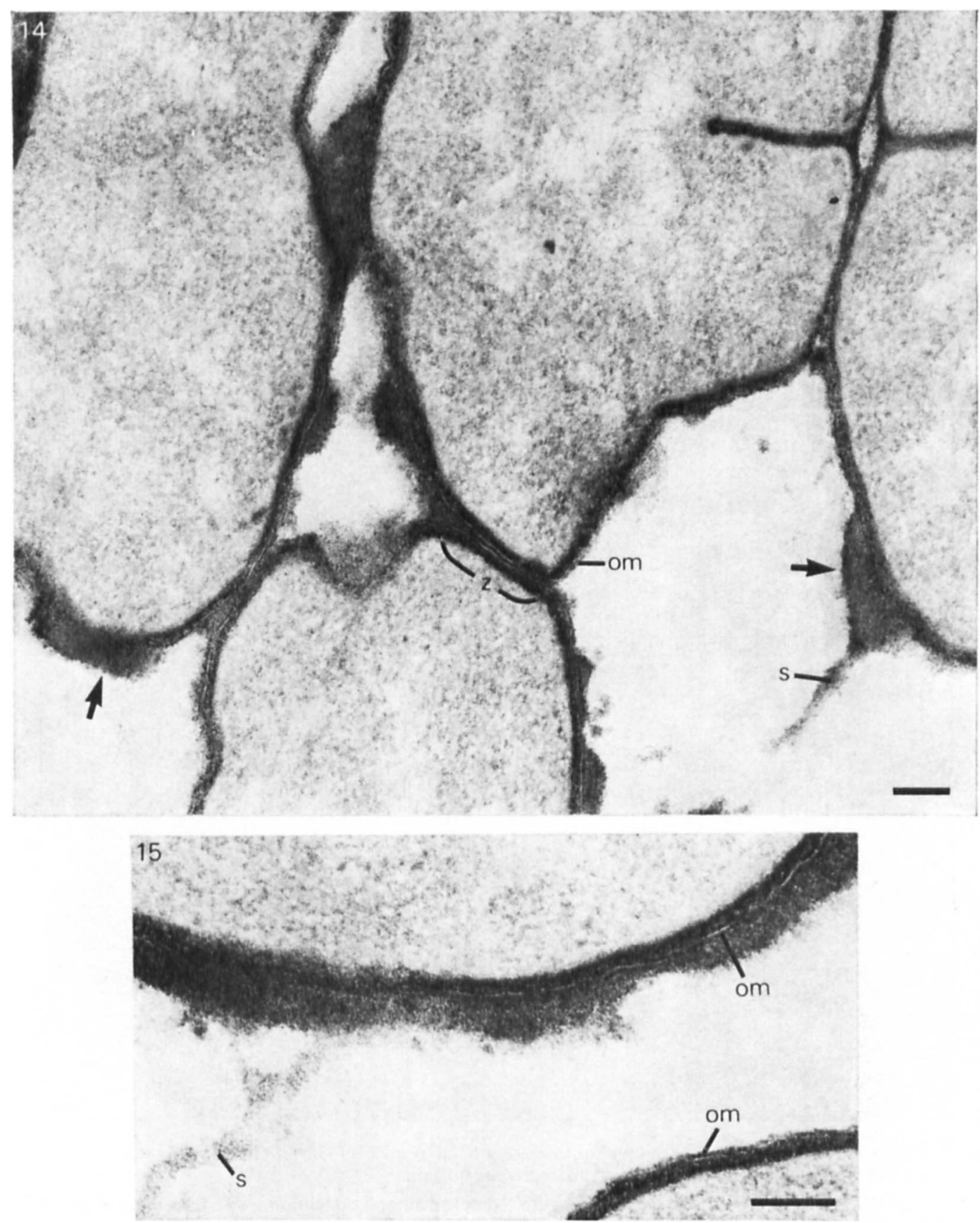

Figs 14, 15. Electron micrographs of thin sections of gonococci (strain Gc12) at the third subculture after growth in vivo. Bar markers represent $0.1 \mu \mathrm{m}$. Alcian blue preparations.

Fig. 14. Irregular masses of dense material adhere to the outer membrane (om) in places (arrows); $\mathrm{s}$, strands of dense material; $\mathrm{z}$, zone of adhesion between cells.

Fig. 15. A few cells have a continuous layer of densely stained material outside the outer membrane (om); s, strand. 

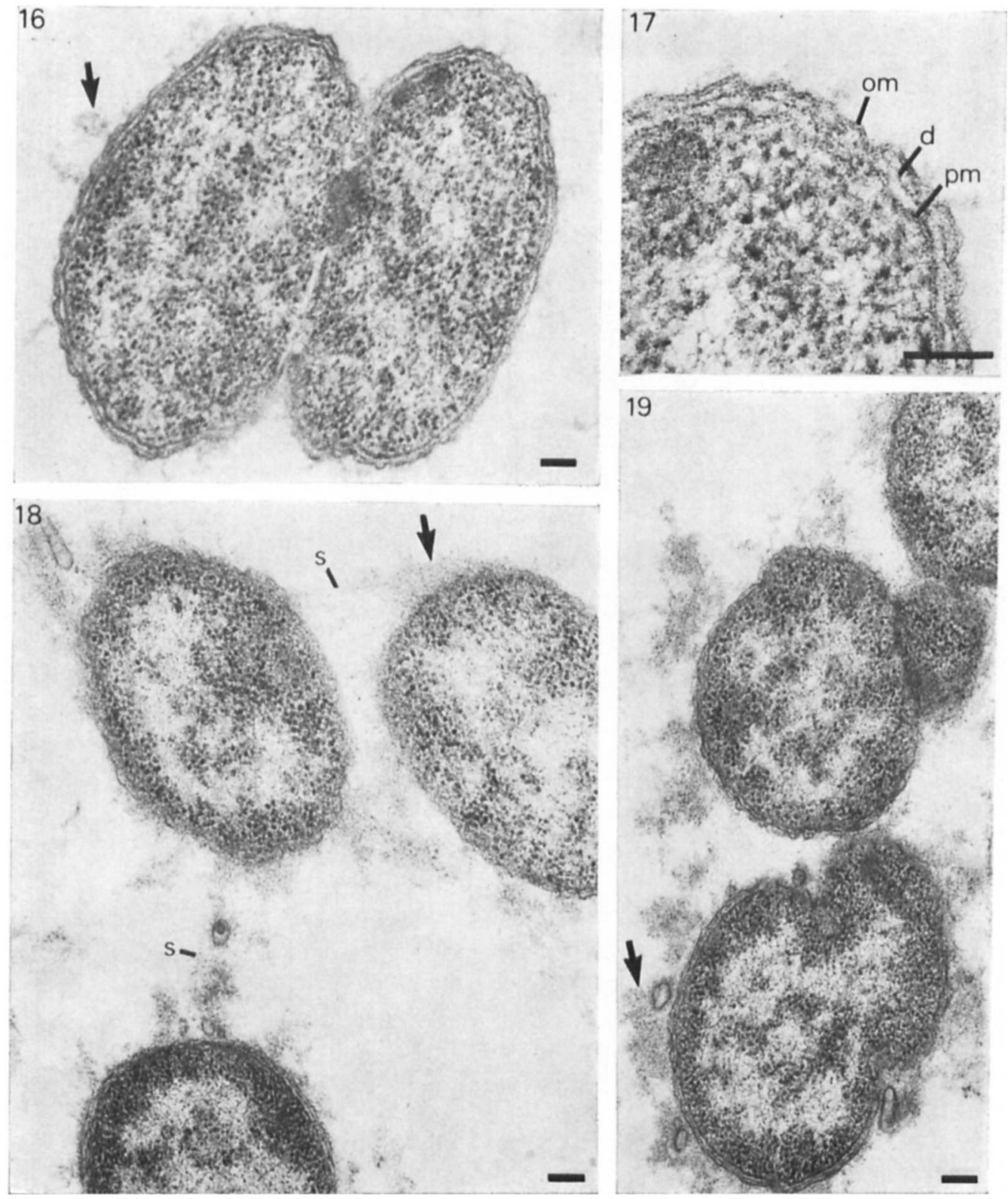

Figs 16 to 19. Electron micrographs of thin sections of gonococci (strain Gc12) at the third subculture after growth in vivo. Bar markers represent $0 \cdot 1 \mu \mathrm{m}$.

Fig. 16. Pre-treated with normal rabbit serum, complement and conglutinin, and then fixed in the normal way; a few small fragments of amorphous material adhere to the outer membrane (arrow).

Fig. 17. Part of Fig. 16 at higher magnification: om, outer membrane; d, dense layer; pm, plasma membrane.

Fig. 18. Pre-treated with antiserum, complement and conglutinin, and then fixed in the normal way; cells are well preserved with no signs of lysis. Amorphous material adheres to the outer surfaces of the cells in irregular masses (arrow) and forms strands (s) between adjoining cells.

Fig. 19. Another portion of the preparation in Fig. 18, with amorphous material adhering to the surfaces of cells (arrow). 
Pre-treatment with antiserum, complement and conglutinin, followed by normal fixation. This treatment was applied to Gc12 only. Since our antiserum was not specific for capsules but was directed against the whole gonococci, it would react with any cytoplasm released by lysis, and therefore great care was taken to avoid lytic effects by using $\mathrm{C}_{6}$-deficient complement for a short period only and by inactivation of the other two sera. The absence of lytic effects is evident from Figs 18 and 19.

The control preparation, with normal rabbit serum, appeared well preserved (Figs 16 and 17) but the layers of the cell envelope were particularly distinct (Fig. 17) and the outer membrane was less convoluted than in bacteria which had not been exposed to serum (Figs 8 and 9). The cell envelope had the same appearance in preparations pre-treated with antiserum, complement and conglutinin (Figs 18 and 19).

A few small fragments of amorphous material adhered to the outer membranes of cells in the control preparation (Fig. 16, arrow), probably because of the very high protein content of the mixture, whilst in the treated preparation irregular masses of amorphous material were seen adjacent to the outer membrane (Fig. 19, arrow). Figure 18 shows similar material (arrow) on the cell surface, apparently connected to strands which stretch between adjoining cells. The similarity in arrangement between the amorphous material forming masses and strands in Figs 18 and 19 and the densely stained surface material of the Alcian blue preparations (Fig. 14) is very marked. The densely stained surface masses and strands are also seen in the Alcian blue preparation of strain Gc40 (Fig. 12).

Comparison of preparations treated with antiserum, complement and conglutinin and those treated with antiserum alone showed little, if any, advantage for the more complicated method used here, and so in future preparations will be made with antiserum only.

\section{DISCUSSION}

The observations on colony type were rather unexpected. It has been reported that the minimum infective dose for guinea-pig chambers of gonococci of types 1 and 2 is less than that of types 3 and 4 (Arko \& Wong, 1977; Veale et al., 1975; Penn et al., 1976), and so it was expected that types 1 and 2 would have a selective advantage during growth in the chamber, and that a mixed inoculum would result in a population consisting predominantly of types 1 and 2. This was not found, even after prolonged infection of guinea-pig chambers.

The results obtained by light microscopy confirm the presence of capsules, as recently reported by Richardson \& Sadoff (1977) and James \& Swanson (1977).

An early study (Fitz-James, 1964), using osmium fixation for the electron microscopy of thin sections of a recently isolated gonococcus, showed fuzzy material adhering to the outer membrane; this was interpreted as fixed medium. In more recent studies, the method has included preliminary fixation with glutaraldehyde, followed by osmium fixation, and sometimes by block staining with uranyl acetate. Such methods did not show any visible material outside the outer membranes when applied to cultures of gonococci (Swanson et al., 1971), to gonococci in subcutaneous chambers in mice (Arko, Bullard \& Duncan, 1976) or to urethral material from male patients (Ward \& Watt, 1972; Ovčinnikov, Delektorskij \& Dmitriev, 1976). However, Novotny, Short \& Walker (1975) showed gonococci with fuzzy external coats inside phagosomes in urethral material, and ruthenium red staining of gonococci grown in culture in ascitic fluid revealed some fuzzy extracellular material (Ovčinnikov et al., 1976). Hendley et al. (1977) treated broth cultures of recently isolated gonococci with homologous antiserum, and found external layers of amorphous material irregularly distributed around the bacteria, which were thought to be capsule combined with antiserum.

Our results with Alcian blue-lanthanum fixation of gonococci of two strains subcultured three times after growth in vivo in the guinea pig clearly show dense material outside the outer membrane. Some cells in the preparations have thick continuous layers of this 
material while others only have irregular masses and strands. It is not known whether the masses arise from disruption of a continuous layer during preparation for microscopy, or whether they represent the actual distribution of extracellular material on gonococci at this stage of growth. It is possible that fixation with Alcian blue of gonococci in the chamber fluid might show more bacteria with continuous capsules.

The penetration of the dense stain through the outer membrane to the surface of the plasma membrane was also observed by Cassone \& Garaci (1974) in E. coli. They used a similar staining technique except that Alcian blue was used in combination with tris(1-aziridinyl)phosphine oxide (TAPO). In our preparations the outer membrane remained clearly visible, and so there is no doubt which part of the densely stained material is extracellular in location.

In an earlier study of lactobacilli in the chicken crop using the Alcian blue-lanthanum method, Brooker \& Fuller (1975) observed the presence of extracellular layers and connecting strands between the bacteria and adjacent areas of the membranes of chicken crop epithelial cells. Similar observations were made by Fletcher \& Floodgate (1973), using Alcian blue alone, on a marine bacterium adhering to Millipore filters.

The Alcian blue-lanthanum method was originally applied to mammalian tissues (Shea, 1971) and the basis of the staining is thought to be the reaction of Alcian blue, a cationic dye, with polyanionic substances followed by enhancement of the contrast by lanthanum (Shea, 1971). For the gonococcus, this would imply that the densely stained outer layer is composed of negatively charged material. Apicella (1974) isolated an acidic polysaccharide from gonococci, but his results did not indicate whether it was derived from the lipopolysaccharide or from capsular material.

The cells treated with antiserum, complement and conglutinin had large amounts of amorphous material in irregular masses adjacent to the outer membrane with occasional strands between adjoining cells, and the similarity in arrangement between this material and the densely stained material in the Alcian blue preparations strongly suggests that both methods are revealing the same extracellular material. Gonococci completely surrounded by extracellular material, such as are seen in Alcian blue preparations, are lacking in the preparations with antiserum, probably because treatment with antiserum involves making a suspension of the bacteria, thus disrupting the capsules. Our results confirm those of Hendley et al. (1977), who used antiserum only.

The strands connecting neighbouring bacteria seen in both the Alcian blue and the antiserum-treated preparations are unlikely to be DNA since they are seen in a DNAasetreated preparation (Fig. 18). It also seems unlikely that they are pili, since the preparations consisted predominantly of large colonies and no traces of pili were seen in the normal glutaraldehyde-osmium preparation. The strands appear continuous with the irregular masses of extracellular material (Figs 12 and 14) or with continuous layers (Fig. 15), and it seems likely that they consist of the same material.

Light and electron microscope observations of gonococci recently grown in vivo differed in that light microscopy revealed capsules on nearly all the gonococci in a preparation, whereas the best electron microscope preparation method, using Alcian blue, showed continuous capsules on only a proportion of the cells. Since the two methods differ in many respects, this is not entirely surprising. In particular, the preparation for electron microscopy entails a long sequence of processes during which capsular material could easily be detached.

We acknowledge the support of the Wellcome Trust (to R.D.H.), the Medical Research Council and the Microbiological Research Establishment (to M.J.T.), and the Sir Halley Stewart Trust (to A.M.G.). We thank the Wellcome Trust for the loan of the AEI EM6B electron microscope. We are grateful to Dr P. J. Lachmann for the gift of $\mathrm{C}_{6}$-deficient rabbit serum, and to R. A. Parker, Jennifer O. Briggs and Janet Atherton for skilled technical assistance. 


\section{REFERENCES}

Apicella, M. A. (1974). Antigenically distinct populations of Neisseria gonorrhoeae: isolation and characterization of the responsible determinants. Journal of Infectious Diseases 130, 619625.

Apicella, M. A. \& Allen, J. C. (1973). Isolation and characterization of the $\beta$ antigen of Neisseria gonorrhoede. Infection and Immunity 7, 315-321.

ArKo, R. J. \& WonG, K. H. (1977). Comparative physical and immunological aspects of the chimpanzee and guinea-pig subcutaneous chamber models of Neisseria gonorrhoeae infection. British Journal of Venereal Diseases 53, 101-105.

Arko, R. J., Bullard, J. C. \& Duncan, W. P. (1976). Effects of laboratory maintenance on the nature of surface reactive antigens of Neisseria gonorrhoeae. British Journal of Venereal Diseases 52, 316-325.

BRoOKer, B. E. \& Fuller, R. (1975). Adhesion of lactobacilli to the chicken crop epithelium. Journal of Ultrastructure Research 52, 21-31.

CASSONE, A. \& GaraCI, E. (1974). Lanthanum staining of the intermediate region of the cell wall in Escherichia coli. Experientia 30, 1230-1231.

Fitz-JAMES, P. (1964). Thin sections of dividing Neisseria gonorrhoeae. Journal of Bacteriology 87, 1477-1482.

Fletcher, M. \& Floodgate, G. D. (1973). An electron-microscopic demonstration of an acidic polysaccharide involved in the adhesion of a marine bacterium to solid surfaces. Journal of General Microbiology 74, 325-334.

Glauert, A. M. \& Thornley, M. J. (1969). The topography of the bacterial cell wall. Annual Review of Microbiology 23, 159-198.

Greaves, R. I. N. (1960). Preservation of living cells by freeze-drying. Annals of the New York Academy of Sciences 85, 723-728.

Hendley, J. O., Powell, K. R., Rodewald, R., Holzgrefe, H. H. \& Lyles, R. (1977). Demonstration of a capsule on Neisseria gonorrhoeae. New England Journal of Medicine 296, 608-611.

James, J. F. \& Swanson, J. (1977). The capsule of the gonococcus. Journal of Experimental Medicine 145, 1082-1086.

Kellogg, D. S., JR, Peacock, W. L., JR, Deacon, W. E., Brown, L. \& Pirkle, C. I. (1963). Neisseria gonorrhoeae. I. Virulence genetically linked to clonal variation. Journal of Bacteriology 85, 12741279.

Novotny, P., Short, J. A. \& Walker, P. D. (1975). An electron-microscope study of naturally occurring and cultured cells of Neisseria gonorrhoeae. Journal of Medical Microbiology 8, 413-427.

Ovčinnikov, N. M., DelektorskiJ, V. V. \& DMITRIEV, G. A. (1976). Ultrastructure of gonococci in acute, chronic and asymptomatic gonorrhoea. British Journal of Venereal Diseases 52, 230-245.

Penn, C. W., Sen, D., Veale, D. R., Parsons, N. J., SMrTh, H. \& WITT, K. (1976). Morphological, biological and antigenic properties of Neisseria gonorrhoeae adapted to growth in guinea-pig subcutaneous chambers. Journal of General Microbiology 97, 35-43.

Richardson, W. P. \& Sadoff, J. C. (1977). Production of a capsule by Neisseria gonorrhoeae. Infection and Immunity 15, 663-664.

SHEA, S. M. (1971). Lanthanum staining of the surface coat of cells. Its enhancement by the use of fixatives containing Alcian blue or cetylpyridinium chloride. Journal of Cell Biology 51, 611-620.

Swanson, J., Kraus, S. J. \& Gotschlich, E. C. (1971). Studies on gonococcus infection. I. Pili and zones of adhesion: their relation to gonococcal growth patterns. Journal of Experimental Medicine 134, 886-906.

Thornley, M. J., Glauert, A. M. \& Sleytr, U. B. (1973). Isolation of outer membranes with an ordered array of surface subunits from Acinetobacter. Journal of Bacteriology 114, 1294-1308.

Veale, D. R., Smith, H., Witt, K. A. \& Marshall, R. B. (1975). Differential ability of colonial types of Neisseria gonorrhoeae to produce infection and an inflammatory response in subcutaneous perforated plastic chambers in guinea-pigs and rabbits. Journal of Medical Microbiology $\mathbf{8}$, 325-335.

WARD, M. E. \& WATT, P. J. (1971). The preservation of gonococci in liquid nitrogen. Journal of Clinical Pathology 24, 122-123.

WARD, M. E. \& WATT, P. J. (1972). Adherence of Neisseria gonorrhoeae to urethral mucosal cells: an electron-microscopic study of human gonorrhea. Journal of Infectious Diseases 126, 601-605. 